\title{
Komplikationen der Leberzirrhose
}

$\mathrm{K}$ omplikationen der Leberzirrhose begegnen uns täglich in der Praxis. Auch wenn die Lebertransplantation die einzige kurative Therapie für die Betroffenen ist, so ist es von vitaler Bedeutung, Komplikationen - wie die Ösophagusvarizenblutung, den therapierefraktären Aszites, die hepatische Enzephalopathie oder das hepatozelluläre Karzinom - rechtzeitig zu erkennen und zu behandeln, denn eine adäquate Therapie verbessert sowohl die Lebensqualität als auch die Lebenserwartung der Patienten.

Unser Verständnis der Pathogenese der portalen Hypertension hat sich in letzter Zeit grundlegend erweitert und verändert, was die Einführung neuer und die Neubewertung alter Therapiestrategien nach sich zog. Durch große klinische Studien wissen wir um die Wirksamkeit verschiedener Pharmaka in der Prävention und Therapie einer akuten Ösophagusvarizenblutung und die Möglichkeit, das individuelle Blutungsrisiko zu quantifizieren. Welchen Stellenwert die einfach durchzuführende Lebervenenverschlussdruckmessung zur Risikostratefizierung und zur Therapiekontrolle hat, fasst PD P. Schiedermaier, Saarbrücken, hier ebenso zusammen, wie risikoadaptierte Therapiestrategien.

Weitere therapeutische Herausforderungen neben der akuten Blutung sind der therapierefraktäre Aszites und das hepatorenale Syndrom. Wer hierbei von einem transjugulären intrahepatischen portosystemischen Stent-Shunt (TIPS) profitiert, ist die Frage, die wir uns im Alltag immer wieder stellen. Herr Prof. M. Rössle, als einer der führenden Experten auf diesem Gebiet stellt hier evidenzbasierte Empfehlungen vor.

Die hepatische Enzephalopathie als weitere Komplikation der Leberirrhose beeinträchtigt die Lebensqualität der Betroffenen schon im frühen Stadium der Erkrankung erheblich. Neue diagnostische Verfahren ermöglichen die Früherkennung und damit eine frühzeitige Therapie dieses Syndroms. Dr. G. Kircheis, Mitarbeiter der Arbeitsgruppe von Professor Häussinger an der Universität Düsseldorf, stellt nicht nur das dort entwickelte, neue diagnostische Verfahren vor, sondern wertet auch kritisch die verschiedenen Therapieansätze.

Leider sehen wir auch immer wieder Patienten mit hepatozellulärem Karzinom auf dem Boden der Leberzirrhose. Der Pionier der Lokalbehandlung mithilfe der Radiothermofrequenzablation in Deutschland, PD H.-P. Allgaier, Titisee-Neustadt, unterzieht dieses und andere lokal therapeutische Verfahren im Kontext der systemischen Therapie einer kritischen Wertung und erläutert ein differenziertes Vorgehen.

Insgesamt zeigen die Beiträge, dass es in letzter Zeit nicht nur zu einem Fortschritt im Verständnis der Genese der Komplikationen der Leberzirrhose gekommen ist. Vielmehr wurden die therapeutischen Konzepte weiterentwickelt, was für den Patienten zum einen eine bessere Lebensqualität aber auch ein längeres Überleben bedeutet - wenn auch die Ergebnisse aller Bemühungen noch hinter den Wünschen und Erwartungen von Arzt und Patienten zurückbleiben.

Wir möchten mit diesem Themenheft anregen, die heutigen diagnostischen und therapeutischen Möglichkeiten für Patienten mit Komplikationen der Leberzirrhose kritisch und bewusst zu überdenken. Gerade durch die Identifizierung des individuellen Blutungsrisikos sowie die differenzierte Indikationsstellung zur TIPS-Implantation bei Patienten mit therapierefraktärem Aszites sind hier deutliche Vorteile für den Patienten zu erwarten.

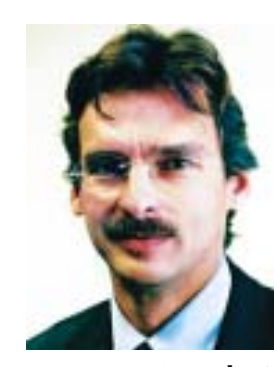

PD Dr. D. Grandt, Saarbrücken (Gasteditor)

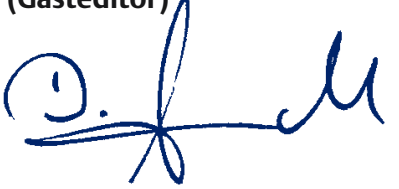

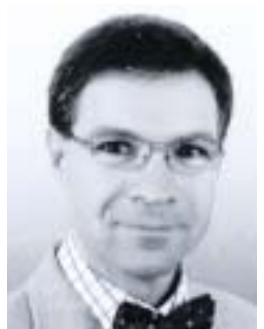

PD Dr. P. Schiedermaier, Saarbrücken (Gasteditor)

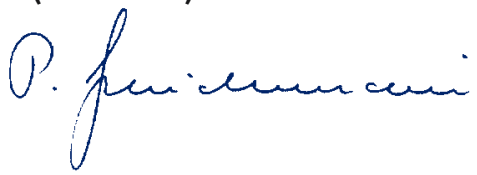

